\title{
Espacios de aventuras en el Libro de Apolonio*
}

\author{
Natacha CROCOLL \\ Université de Genève, Suiza \\ natacha.crocoll@unige.ch
}

El Libro de Apolonio es un texto donde la dimensión espacial cobra un protagonismo inédito en la construcción del discurso, en comparación con las demás obras del siglo XIII (con la notable excepción del Libro de Alexandre). Ahora bien, este aspecto de la obra no parece haber sido objeto de especial atención entre los críticos que han trabajado sobre ella. Contamos con algunas páginas en los estudios de Manuel Alvar (1976: 151-161), de Alan Deyermond (1989: 153-164) y de Joaquín Artiles (1976: 35-43), así como ciertos apuntes en el prólogo a la edición de Dolores Corbella (2007: 32-41). Unos comentarios sobre el papel del mar y de la ciudad -por Diana Berruezo Sánchez (2012: 381-386), Alberto Navarro González (1962: 242-262) y, sobre todo, Rafael Rodríguez Victoria (2019: 71-84) - y otros sobre la función del viaje en la obra -por ejemplo, Matthew Desing (2011: 1-15) y Olivier Biaggini (2007: 38-40)- completan este panorama. Hace falta, pues, proponer una lectura de conjunto que analice los fragmentos espaciales de la obra y su papel dentro del discurso poético ${ }^{1}$.

Para llevar a cabo este propósito, el presente artículo se enfocará, primero, en la función estructural de los dos espacios principales presentes en el Libro, a saber, el mar y las ciudades. Una segunda parte girará en torno a la importancia simbólica de ciertos fragmentos descriptivos que no se limitan a meras «indicaciones topográficas o puramente locativas», para retomar una formulación de Joaquín Artiles (1976: 36-37), sino que participan de las pruebas y de la trayectoria de los distintos personajes. Finalmente, se analizarán algunos versos que el autor anónimo del Apolonio ha convertido en verdaderos paisajes y se examinará, a raíz

* Este trabajo ha sido elaborado dentro del proyecto Doc.mobility: La fonction du paysage dans la littérature castillane du Moyen Âge (XIII -XVe s.) (id. P1GEP1_187827), financiado por el Fonds National Suisse de la Recherche Scientifique.

${ }^{1}$ El objetivo de este artículo se encuentra, por consiguiente, a medio camino entre la geocrítica -el estudio de la construcción del espacio literario en sus dimensiones estructural y de contenido- y la geopoética - el análisis de las formas estilísticas que sustentan dicha construcción (Collot 2014). Más precisamente, sigue las pautas de investigación propuestas por Ribémont (2003: 47) para la elaboración de una geocrítica de la literatura medieval, a saber, el análisis de los distintos elementos que componen el complejo y «laminado» espacio literario de los siglos medios, así como su proyección sobre la recepción de los textos. 
de los extractos traídos a colación, el cuidado particular con el que ha sido elaborado el espacio de la versión castellana.

\section{LA FUNCIÓN ESTRUCTURAL DEL ESPACIO: UN VIAJE ENTRE MAR Y CIUDAD}

El Libro de Apolonio es, ante todo, un libro de viajes; el héroe y su familia se encuentran, por turnos, en no menos de seis ciudades ubicadas en el Mediterráneo oriental (Tiro, Antioquía, Tarso, Pentápolis, Éfeso y Mitilene). En cada una de ellas, los distintos protagonistas se ven sometidos a prueba de una manera $\mathrm{u}$ otra, marcando etapas en su desarrollo personal. Si en Mitilene, por ejemplo, se prueba la virtud de Tarsiana, que debe encontrar un medio de ganarse la vida para escapar de la prostitución, es la trayectoria urbana de Apolonio la que mejor refleja esta iniciación que lo llevará a establecerse como modelo de actuación para el público del siglo XIII (Berruezo Sánchez 2012: 380)². Para empezar, en Antioquía, el personaje debe contestar a las adivinanzas del rey incestuoso y se confronta con los límites del saber que adquirió a través de sus libros ${ }^{3}$. Después de ello, Apolonio llega a Pentápolis despojado de sus atributos reales por un naufragio y se gana el amor de Luciana por su cortesía y su habilidad de músico cortés, y así sucesivamente.

Más allá de esta dimensión formativa de la urbe, Rafael Rodríguez Victoria (2019) analiza las relaciones que establece el protagonista con las ciudades que atraviesa en el marco de la negociación de poderes que experimenta Castilla en el siglo XIII entre la autoridad real y los centros urbanos ${ }^{4}$. Para el investigador, «todas las ciudades del relato están representadas con una finalidad aleccionadora, que se corresponde con la legitimación del sistema monárquico como sistema de gobierno ideal» (Rodríguez Victoria 2019: 82). Así, Apolonio, convertido en rey modélico gracias a sus peripecias, sale ganador de sus interacciones con las distintas autoridades de las ciudades que visita (con la excepción de Éfeso): hereda, por ejemplo, la corona de Pentápolis gracias a su boda con Luciana y la de Antioquía por haber resuelto el enigma y haber sido elegido por los notables de la corte de su antiguo enemigo. En definitiva, «desde la perspectiva monárquica del poema, la ciudad puede ser un lugar seguro y próspero, en beneficio del reino, siempre y cuando sea dirigido y ordenado por aquel que encarna las virtudes humanas más altas: Apolonio, el rey» (idem).

${ }^{2}$ Sobre Apolonio y el nuevo papel de las ciudades en el siglo XIII, véase Manuel Alvar (1984: 63-65).

${ }^{3}$ Solo el conocimiento mezclado con la experiencia puede llevar al saber según Diana Berruezo Sánchez (2012: 380). A su vez, Desing (2011: 9) expresa algo similar al distinguir entre sapientia (saber libresco) y fortitudo (conocimiento puesto en acción).

${ }^{4}$ El artículo de Rafael Rodríguez se inspira del trabajo de Julian Weiss (2006: 198-209). 
Ahora bien, a pesar de su función esencial como núcleos de acción dentro de la narración, las ciudades aparecen apenas esbozadas en la fuente latina del texto, la Historia Apollonii regis Tyri. En cambio, aunque escasean las largas descripciones topográficas en la versión castellana, sí que contamos en ella con más detalles. Consideremos, a modo de ejemplo, el mercado de Pentápolis, donde Apolonio deambula con su futuro suegro. En el párrafo XIX del texto latino, según la transcripción de Manuel Alvar (1976: 157), consta únicamente lo siguiente: «Rex autem post poces dies tenens Apolonio manu forum petit et cum eo deambulauit». Por el contrario, el poeta castellano desarrolla la escena y la adapta a su tiempo, adecuando para ello el fórum de la época a la realidad del siglo XIII ${ }^{5}$ :

Ouo sabor hun día el rey de caualgar, andar por el mercado, ribera de la mar; fizo ha Apolonio, su amigo, llamar, rógole que sallyese con él ha deportar.

Prísolo por la mano, non lo querìa mal, vieron por la ribera mucho buen menestral: burzeses et burzesas, mucha buena senyall, salieron al mercado, fuera al reyal.

(cc. 201-202) $)^{6}$

Como se puede notar, la ubicación en el arenal y los ciudadanos que pasean son novedades de la versión castellana, inspiradas en la cotidianidad de una ciudad mercantil situada en la ribera del mar (Alvar 1976: 157-158). Para Rodríguez Victoria (2019: 81), además, la descripción del mercado y de la gente que circula por él da fe de la prosperidad y estabilidad del reino de Pentápolis gracias «al buen gobierno de Architrastres, rey virtuoso y sabio, que continuará administrando, una vez incorporada la ciudad al territorio de Apolonio, el hijo de éste y de Luciana».

El otro gran núcleo espacial que estructura la obra es el Mediterráneo $^{7}$, sobre cuyo papel fundamental se hace énfasis en las adivinanzas de Tarsiana, que giran en su mayoría alrededor de los temas del agua y del mar (González Vera 2001: 17). El espacio marítimo, en la literatura medieval en general, es polifacético y puede desempeñar funciones múltiples y hasta contrarias: la muerte, la vida, el viaje y la providencia son tan solo algunas de ellas (Lebrero Cocho 2015: 276). Aunque no sea el propósito de este artículo enumerar sus múltiples posibilidades, es

${ }^{5}$ Un ejemplo similar de medievalización es, por supuesto, la transformación del templo de Artemis en monasterio, donde se va a recluir Luciana a la espera de su marido (c. 324).

${ }^{6}$ Todas las citas corresponden a la edición de Dolores Corbella (2007).

${ }^{7}$ Según el cómputo de Deyermond (1989: 157), la palabra mar aparece no menos de treinta y siete veces en el texto y su sinónimo, ondas, diez. 
importante resaltar, en suma, sus diversos usos en la construcción de un espacio de aventuras y (des)encuentros a lo largo de la tradición literaria del Medievo ${ }^{8}$.

En el Libro de Apolonio, el mar cumple, ante todo, una función estructural, dado que sirve para articular los distintos episodios de las empresas de los protagonistas: : "Rather than an isolated instance, the image of the [sea] journey as a space for the disordering and reordering of meaning is a more generalized theme throughout the poem» (Desing 2011: 6). En otras palabras, permite enlazar las diferentes peripecias de la obra al llevar a sus personajes de una aventura a la otra y al marcar su evolución individual ${ }^{10}$.

Una clara muestra de dicha función estructural lo proporciona la comparación de dos tempestades en el Apolonio: la primera, cuando el héroe deja Tarso y ocurre su primer naufragio y, la segunda, cuando la providencia procura acercarlo a Tarsiana hacia el final de la obra. Los fragmentos son los siguientes:

\section{cc. $107-110$}

El mar, que nunqua touo leyaltat ni belmez, cámiase priuado et ensányase rafez, suele dar mala çaga, más negra que la pez: el rey Apolonio cayó en essa vez.

Quanto tenién dos horas, abez auìan andado, boluiéronse los vientos, el mar fue conturbado, nadauan las arenas, el çiello leuantando, non auié hi marinero que non fuese conturbado. Non les valién las áncoras, que non podién trauar, los que eran maestros non podién gouernar, alçáuanse las naues, querìanse trastornar, tanto que ellos mismos non se sabién conseiar. Cuytóles la tempesta et el mal temprao, perdieron el conseio et el gouierno capdal, los árboles de medio todos fueron a mal, iguárdenos de tal cuyta el Seyor Espirital!

\section{cc. $453-456$}

Fueron luego las áncoras a las naues tiradas, los rimos aguisados, las velas efestadas; tenién viento bueno, las ondas bien pagadas, fueron de la ribera aýna alongadas.

Bien la media carrera o más hauién andada, auian sabrosos vientos, la mar iazié pagada; fue en poco de rato toda la cosa camiada, tollióles la carrera que tenién començada. De guisa fue rebuelta et yrada la mar, que non auién nengún conseio de guiar; el poder del gouernyo houiéronlo ha desemparar, non cuidaron ningunos de la muerte escapar. Prísolos la tempesta et el mal temporal, sacólos de caminos el oratge mortal, écholos su uentura et el Rey Espirital en la vila que Tarsiana pasaua mucho mal.

Como apunta Joaquín Artiles (1976: 42), se puede apreciar un fuerte paralelismo, o incluso se podría decir una repetición entre ambas tempestades. Por una parte, en las dos se insiste en el carácter tramposo del mar que, pese a su aparente calma inicial, empieza pronto a agitarse (vv. $107 \mathrm{ab}$ y c. 454) y conlleva a la pérdida de control sobre partes clave del

\footnotetext{
${ }^{8}$ Para un abanico más completo de las mencionadas funciones, consúltese la monografía de Alberto Navarro González (1962).

${ }^{9}$ Diana Berruezo Sánchez (2012: 381) comparte esta interpretación y se refiere al mar como «elemento dúctil, sinuoso y cambiante».

${ }^{10} \mathrm{Al}$ solo personaje de Apolonio se le pueden atribuir trece travesías, de las cuales cuatro aparecen sin que se mencione el mar, otras cuatro con breve alusión a este espacio y las cinco restantes ofrecen una mayor «valorización del elemento marino» (Artiles 1976: 39), sobre la que volveremos a continuación.
} 
barco, lo que provoca, a su vez, el cambio de rumbo de la nave. Por otra parte, el mar aparece claramente personificado en ambos fragmentos; ello se puede observar, por ejemplo, con la mención de la «deslealtad del mar» (v. 107a) y de su ira (v. 455a), convirtiéndolo en un verdadero antagonista del personaje. El paralelismo se construye también a nivel léxico, dado que varias palabras se repiten, aunque en distinto orden ${ }^{11}$. Finalmente, el nudo de la tempestad es desarrollado en exactamente el mismo número de estrofas y se puede notar una repetición casi exacta entre los versos 110a y 456a, así como una clara similitud en las formulaciones para referirse a Dios.

Por supuesto, la redundancia entre ambos extractos no es casual. Es posible que el poeta castellano solo repitiera aquí un tópico literario ${ }^{12}$, pero llama la atención el lugar del relato donde intervienen estas tormentas. La primera provoca la acción y permite que Apolonio se despoje de todo su pasado para emprender su camino iniciático hacia la virtud y la experiencia; corresponde al momento donde empieza realmente la aventura. La segunda marca otro momento clave: el comienzo de los reencuentros del rey con su familia, después de las peripecias que les permitieron fortalecerse y establecerse como modelos de moral y de comportamiento. Las tempestades señalan, por consiguiente, dos hitos o dos ejes que separan los grandes bloques temáticos del relato y muestran el giro de la fortuna para los personajes.

\section{Dimensión SIMBÓliCA DEL ESPACIO EN EL LIBRO DE APOLONIO}

Asimismo, estrechamente vinculada con la dimensión estructural del mar y la función de las tempestades, surge la noción de peregrinatio vitae. Como es bien sabido, el motivo de la vida humana como un peregrinaje es uno de los más extendidos en la literatura del Medievo y encuentra sus raíces en el tópico de la búsqueda del paraíso perdido ${ }^{13}$. De hecho, el exilio del hombre del Edén señala una forma de expiación por la culpa de Adán y Eva, culpa que aliena al Hombre de la divinidad y lo obliga a sufrir en la tierra antes de poder recuperar -o no- la gracia inicial (Biaggini 2007: 27). En cuanto al Libro de Apolonio, el

${ }^{11}$ Es el caso de «áncoras» (vv. 109 y 453a), «vientos» (vv.108 y 454b), «conseiar/conseio» (vv. 109d y 455c) y «gouernar/gouierno/gouernyo» (vv. 109b, 110b y 455c).

${ }^{12}$ La importancia otorgada a las tempestades en el Libro de Apolonio no es propia de la versión castellana, sino que resulta ser un rastro heredado de la novela griega. De hecho, Pablo Ancos (2018: 285) afirma que ambas tienen en común los múltiples movimientos del héroe, «las separaciones bruscas y las anagnórisis repentinas, los peligros y las persecuciones, los naufragios o las falsas muertes». A su vez, Deyermond (1989: 157) hace énfasis en el protagonismo del mar y de las tempestades en la ficción helenística, aún aumentado en la versión latina y en sus traducciones a lenguas vernáculas.

${ }^{13}$ El motivo se encuentra en la Biblia, por ejemplo, en Sal. 119, 19; Pe. 2, 11; Heb. 11, 13; y Cor. 5, 6-8. 
movimiento constante generado por los viajes facilita la modificación de las circunstancias de los personajes, que puede leerse en el siglo XIII como una alegoría de las penas del hombre en el mundo terrenal (Desing 2011: 6). Así, en palabras de Olivier Biaggini (2007: 39-40):

Apolonio n'est pas seulement un homo viator: il est devenu l'incarnation du topos lui-même, le modèle de cette condition humaine sur laquelle s'acharnent les caprices de la vie de ce monde. [...] Exemple suprême des gains et des pertes alternés, le parcours d'Apolonio n'est pas seulement un désordre, il est l'ordre du monde: en prendre conscience, c'est déjà retrouver une part de son identité. La pérégrination en ce monde, conséquence du péché des hommes, est aussi la seule manière qu'ils ont de se construire une identité renouvelée.

Como señala el investigador francés, el ejemplo por antonomasia de esta dinámica redentora es, por supuesto, el mismo Apolonio, frecuentemente acompañado por el epíteto de peregrino en la versión castellana ${ }^{14}$. Es más, el protagonista asume explícitamente el papel de peregrino en dos episodios clave: el primero es su cambio de apariencia después de la supuesta muerte de Luciana, cuando promete dejar crecer su barba y no cortarse las uñas hasta la boda de su hija - una transformación física claramente vinculada con la idea de alienación y arrepentimiento-, y el segundo consiste en su partida a Egipto, «tierra emblemática de penitencia» después de creer en la muerte de Tarsiana (Cuesta Torre 1999: 12).

En definitiva,

Reinterpretando los continuos viajes de Apolonio desde esta perspectiva, el lector medieval se encontraba con un continuo peregrinaje del personaje, para el cual el «mar» puede ser arquetipo de la dinámica de la vida, del exilio voluntario, pero también de la vida entendida como trayecto hacia un más allá (Corbella 2007: 34).

Además de este papel del mar como cauce de la peregrinatio vitae, se les añaden a las ondas otros valores simbólicos. Uno de ellos es el de la fortuna o de la providencia ${ }^{15}$, que convierte el espacio marítimo en una entidad «benigna o adversa, amiga y enemiga al mismo tiempo» (Berruezo Sánchez 2012: 383), cuyo aspecto cambiante denuncia Apolonio en la copla 120: «Nunqua deuìa omne en las mares fiar, / traen

${ }^{14}$ Véase el artículo de Marina Scordilis Brownlee (1983: 169-170) para la enumeración de las ocasiones en las que se identifica a Apolonio como peregrino. En cambio, la estudiosa apunta que, en la versión latina, la palabra peregrinus solo aparece dos veces, hecho que interpreta como prueba de la mayor cristianización del texto del siglo XIII.

${ }^{15}$ Aparece fortuna, como sinónimo de suerte, por ejemplo en la copla 266: «Auìan de la marina gran partida andada, / podién auer aýna la mar atrauesada, / tóuoles la ventura huna mala çellada, / qual nunca fue ha omnes otra peyor echada». Sobre el papel de la fortuna en el Apolonio y sus fuentes, véanse Manuel Alvar (1976: 50) y Diana Berruezo Sánchez (2012: 383). 
lealtat poca, seben mal solazar, / saben al reçibir buena cara mostrar, / dan con omne aýna dentro en mal logar».

Como se acaba de demostrar, este aspecto encuentra su mayor expresión en las tempestades que alteran por completo la vida de los distintos personajes (causa del naufragio de Apolonio en Pentápolis, de la separación de los esposos y del último reencuentro familiar) y representan, por lo tanto, los giros que puede tomar el destino del hombre (Biaggini 2007: 32). En este sentido, es de notar que, en la versión castellana, los viajes del protagonista suelen acabar mal cuando son motivados por la huida o la vanidad (por ejemplo, cuando Apolonio quiere ceñirse la corona de Antioquía), pero, por el contrario, se vuelven exitosos cuando los desplazamientos son guiados por la penitencia (su huida a Egipto) o por Dios: es el caso del episodio de la aparición del ángel que lo manda sin incidentes primero a Éfeso y, luego, a Tarso. El éxito de estas últimas travesías, y la superación por el héroe de nuevas peripecias que culminan en la anagnórisis final, demuestran que Apolonio ha logrado la ayuda divina en su propia trayectoria vital (Zubillaga 2015: 437).

Además de esta connotación providencial, a veces se adivina cierta dimensión bautismal en la función del mar. El ejemplo más claro de ello es por supuesto el de Luciana, la esposa que lanzan al mar después de su supuesta muerte en el parto. El lector sigue el ataúd de la reina hasta Éfeso donde, gracias al cuidado del discípulo de un médico, vuelve a la vida después de tres días, en clara referencia a Cristo, y emprende una vida monacal hasta que vuelva su esposo, experimentando como un despertar hacia la vida espiritual.

Otros personajes van a vivir una situación similar, como Apolonio, que sale renovado de su naufragio (antes de llegar a Pentápolis), ya que aparece despojado de todos sus privilegios, «trasformado de rey en mendigo y de orgulloso en humilde» para abrir paso a su conversión en monarca y hombre modélicos (Cuesta Torre 1999: 11-12). Finalmente, Tarsiana deja su vida de lujo para convertirse en juglaresa después de atravesar el mar en la nave de sus secuestradores ${ }^{16}$. En suma, todos los personajes principales del relato experimentan, después de un viaje marítimo más o menos rocambolesco, un nuevo tipo de vida que los pone a prueba y les permite salir adelante más virtuosos de lo que eran (Gómez Redondo 2009: 165).

Con todo, los espacios marítimos no son los únicos sobre los que se puede verter una luz simbólica. Así, algunos elementos urbanos también dejan vislumbrar un segundo grado de lectura que apoya el desarrollo de la acción narrativa. Un ejemplo de ello es la caracterización de las urbes en función del valor que se quiere atribuir a los personajes que

${ }^{16}$ Nótese, asimismo, que la función de (re)nacimiento desempeñada por el mar resulta particularmente claro en el caso de Tarsiana, que ve la luz en las ondas. 
las dirigen. De esta manera, al comienzo de la obra, Apolonio llega a Antioquía para pedir la mano de la hija de un rey que aparece caracterizado negativamente desde los inicios del texto. En estas primeras líneas, se establece entre los dos monarcas una dicotomía que subraya su oposición moral: por un lado, Antíoco el malo y, por el otro, Apolonio el bueno (Corbella 2007: 41). El poeta lleva la oposición más allá; mientras que Antíoco solo manda construir naves de guerra y «bastirlas de poderes, de armas et de çiuera» (v. 60c), los barcos de Apolonio son embarcaciones de paz llenas de comida y de bienes mercantiles: «de pan e de tesoro mandó mucho cargar» (v. 34c) (Artiles 1976: 43). Más interesante aún, aunque solo sea una hipótesis de lectura, es el hecho de que este maniqueísmo se vuelve a encontrar en algunos detalles de la construcción espacial del relato. De hecho, si las murallas de Antioquía están ornadas de las cabezas de los pretendientes que no lograron encontrar la respuesta a la adivinanza real, «auían muchos por aquesto las cabeças cortadas, / sedían sobre las puertas de las almenas colgadas» (vv. 16ab), la actitud de Apolonio al llegar a Tarso y salvarla de la hambruna es muy distinta, ya que promociona la construcción de murallas como símbolo de protección ${ }^{17}$ :

Dáruoslo [el trigo] he a conpra, pero de buen mercado, como valié en Tiro do lo houe comprado.

Demás, el precio todo, quante fuere llegado, para la cerqua de la villa quiero que seya dado.

Otro elemento urbano de posible vertiente simbólica es el aislamiento geográfico del monasterio donde Luciana espera a su esposo. En efecto, en las instrucciones que le da el ángel a Apolonio para que pueda reunirse con su mujer, le proporciona información sobre la ubicación del edificio: «Demanda por el tempo que dizen de Diana, / fuera yaze de la villa, en huna buena plana» (vv. 579ab). Se ha expuesto que, después de haber sido lanzada al mar, Luciana es el personaje que menos aparece en la serie de peripecias en la que se desenvuelve la familia después de su separación. El ingreso de Luciana en un monasterio aislado «fasta que

\footnotetext{
${ }^{17}$ Rodríguez Victoria (2019: 77) también señala la dimensión simbólica de las veleidades constructoras de Apolonio, pero las subordina a su lectura del asentamiento del poder monárquico sobre los centros urbanos en el siglo XIII, ya que, con esta acción, Apolonio se asegura del aprecio y amor de la ciudad de Tarso, «como elemento importante en la cohesión del vínculo vasallático». A su vez, Ancos (2018: 291-292) lee el episodio como una prueba de la cortesía de Apolonio, expresada a través de su generosidad, dado que sus acciones permiten «subsanar la carestía de la ciudad y compensar por el riesgo que supone dar cobijo a alguien por cuya cabeza el rey Antíoco ha puesto precio [...]», además de ennoblecer la urbe. De ser así, el paralelo entre los dos monarcas que se acaba de indicar en el cuerpo del texto resultaría incluso más evidente, dado que se volverían a vincular las murallas con la violencia de los descabezamientos ordenados por Antíoco.
} 
Dios quisisere que venga su senyor» (v. 324c) se puede, por consiguiente, interpretar como una expresión más, física esta vez, del alejamiento de Luciana de toda vida política y cortesana hasta la vuelta de Apolonio. En otras palabras, la trayectoria de este personaje femenino se deja en suspenso ya que, como queda dicho, las ciudades son el núcleo de la acción narrativa y de la evolución de los personajes; al mantenerse al margen de la urbe de Éfeso, es como si Luciana se viera excluida, provisionalmente, de la historia narrada.

\section{PAISAJES Y ESPACIOS POÉTICOS DE LA VERSIÓN CASTELLANA}

Todavía en las afueras de Éfeso «se adivina [...] [otro] paisaje concreto, pictórico, con horizonte de montañas». Estas palabras, sacadas de un estudio de Joaquín Artiles (1976: 36-37), vienen a matizar la afirmación central del mismo trabajo, a saber, que «el paisaje de Apolonio se reduce, como en el Mio Cid, a simples indicaciones topográficas, puramente locativas, sin propósito poético [...]». Dicho matiz consiste en la recuperación de tres descripciones que Artiles califica de verdaderos paisajes literarios ${ }^{18}$. Uno de ellos es la ya citada ubicación del templo donde se refugia Luciana, «en huna buena plana» (v. 579b).

Artiles identifica otro paisaje en la descripción de la morada del médico cuyo discípulo salva a la esposa de Apolonio (cc. 285 y 286):

Por beuir más viçioso et seyer más a su placer, como fuera de las ruuas biue omne meior, auìa sus aueres do era morador, en ribera del agua, los montes en derredor.

Andaua por la ribera a sabor de el viento, de buenos escolanos trahiya más de çiento, fallaron esta obra de grant engludimiento, que non fizo en ella el agua nuyll enozimiento.

La ubicación de la acción narrativa en riberas del mar es frecuente en el Libro de Apolonio, donde todo ocurre en ciudades porteñas. Ahora bien, este fragmento se destaca entre los otros porque la descripción incluye varios detalles topográficos y subjetivos para elaborar un

\footnotetext{
${ }^{18}$ Aunque se haya sentado un consenso crítico según el que el paisaje, en su plenitud conceptual, nace en el siglo XVII o incluso en el XIX, varios estudiosos han propuesto extender el uso del vocablo paisaje a épocas anteriores, siempre que se maneje con las debidas precauciones terminológicas y metodológicas. Véanse, por ejemplo, los artículos de María Teresa Rodríguez Bote (2014), Natacha Crocoll (2018 y 2019) y, sobre todo, Serge Briffaud (1998), además de la monografía de Raffaele Milani (2007: 61). Siguiendo esta propuesta crítica, en las líneas siguientes, se empleará la fórmula de paisaje literario para designar a aquellas construcciones espaciales -con referencias geográficas reales o no- elaboradas por la voz enunciativa sobre la base de recursos descriptivos que llaman al lector-oyente a representarse un conjunto espacial.
} 
espacio global. Así, empieza con la oposición entre la ciudad como foco de tensión y los lugares externos, donde el médico vive «más viçioso» ${ }^{19}$. Los tres versos que siguen son los que Artiles califica de paisaje «pictórico», dado que describen los entornos de la morada del médico entre mar y montañas y que la alusión al viento añade un elemento de deleite sensible que dinamiza el cuadro al hacerlo más vívido. Finalmente, es importante resaltar el hecho de que todos estos componentes espaciales son una amplificatio propia de la versión castellana, que otorga a la escena «un marco adecuado y verosímil para el descubrimiento del ataúd varado en la arena» (Cuesta Torre 1999: 18) ${ }^{20}$.

Mientras Luciana y el médico se complacen en quedarse fuera del marco urbano, Tarsiana se ve obligada a sobrevivir en el ámbito de la ciudad de Mitilene, situada en la isla de Lesbos. Después de su rapto por piratas y de su venta a un proxeneta local, la joven hija de Apolonio logra salvarse de la prostitución cuando propone vender su talento en vez de su cuerpo. Así, desempeña durante un tiempo el papel de juglaresa y expone sus capacidades musicales, heredadas de su padre, en la plaza de la ciudad. Este fragmento del relato, desarrollado entre las coplas 426 y 429, es otro ejemplo de amplificatio de la versión castellana ya que, en la Historia Apollonii regis Tyri, se trata de un espacio indefinido ${ }^{21}$. En cambio, en el Apolonio, se desarrolla el episodio con su ubicación explícita en un mercado (v. 426d) y la descripción de la repartición de los oyentes en la plaza -que da un indicio, a su vez, de la distribución de la misma-: «finchiénse de omes apriesa los portales, / non les cabién en las plaças, subiénse a los poyales» (vv. 427cd).

Aunque resulta difícil calificar estos dos últimos versos de paisaje, sí son un ejemplo más del cuidado con el que ha sido construido el espacio en el texto castellano frente a los silencios de su fuente. Aquí los detalles añadidos no parecen seguir otro motivo que el de otorgarle vivacidad al espectáculo de Tarsiana. No obstante, también se podrían interpretar dentro de la trayectoria probatoria de la princesa, ya que este

${ }^{19}$ Esta dicotomía es frecuente en la literatura medieval, como se nota, por ejemplo, en varios textos hagiográficos castellanos del siglo xIII, como la Vida de San Millán y la Vida de Santo Domingo de Silos, donde se opone la ciudad tentadora y el yermo ascético, a imagen de la estancia de Cristo en el desierto (Biglieri 2003: 539-553).

${ }^{20}$ Joaquín Artiles (1976: 37) añade a estos dos paisajes del monasterio y de la choza la copla 633 («Mandól' luego dar honrradas vestiduras, / seruientes et servientas et buenas caualgaduras; / de campos et de vinyas muchas grandes anchuras, / montanyas et ganados et muy grands pasturas»), que concibe como un ejemplo de paisaje rural. Sin embargo, se puede objetar que, al tratarse de la recompensa que Apolonio da al pescador de Pentápolis que lo ayudó después de su naufragio, los últimos versos de la estrofa no corresponden a una descripción o a una evocación espacial organizada, sino que se trata de una lista de regalos, razón por la que no queda incluida en el corpus del presente artículo.

${ }^{21}$ En el texto latino, apenas se mencionan la orden de poner bancos en un lugar frecuentado y la masa que viene a escuchar a Tarsiana; la descripción es, pues, menos detallada que en la versión castellana. 
episodio tiene un fuerte eco con las coplas 178-190 en las que Apolonio, desprovisto de todos sus atributos reales, toca música en público en la corte del rey Architrastres, episodio que resalta la dureza de la caída del personaje. Así, «the courtly echoes found in the market space reinforce the sense of Tarsiana's social degradation, and allow for a reading of the scene as an inversion of the earlier courtly performances» (Pascual Argente 2010: 142-143) 22 .

\section{CONCLUSIÓN}

En definitiva, queda establecido que el espacio del Libro de Apolonio dista mucho de limitarse a unas cuantas indicaciones topográficas. Por el contrario, son numerosos los fragmentos en los que, aunque sea con un solo verso, se elabora una descripción que aviva el ambiente. Es el caso de las escenas que ocurren en mercados urbanos (en Pentápolis y en Mitilene), donde el espacio a menudo indefinido de la Historia Apollonii regis Tyri es elaborado con más detalles y adaptado al público del siglo XIII en la versión castellana.

Tampoco se puede defender la ausencia de «propósito poético» (Artiles 1976: 35) en los distintos fragmentos espaciales traídos a colación en el presente artículo. Por une parte, se han citado algunos versos en los que se desarrolla un verdadero cuadro de paisaje, construido por ejemplo alrededor de la casa del médico de Éfeso, donde intervienen lo subjetivo y lo pictórico, como admitió el mismo Artiles.

Por otra parte, varios espacios de la obra distan mucho de ser un mero telón de fondo, puesto que desempeñan una función discursiva propia. Es el caso del mar, cuyo papel es el de enlazar los distintos núcleos urbanos que acompañan la trayectoria de elevación moral de los personajes, a la vez que se le suma a menudo algún sentido más profundo, simbólico, que tiene importancia en el desarrollo de los protagonistas, como la función de la resurrección o de la peregrinatio vitae.

Así, por sus varias funciones -simbólicas, estructurales y, casi se podría decir, estéticas-, el espacio del Libro de Apolonio resulta ser un eje de análisis que llama a estudios de profundización de la cuestión, puesto que aún faltan por explorar los múltiples matices de este texto y de su trayectoria.

${ }^{22} \mathrm{Al}$ respecto, véase también Carlos Alvar (1989). 
Alvar, Carlos (1989), «De Apolo a Orfeo. A propósito del Libro de Apolonio», Vox Romanica, 48, pp. 165-172.

Alvar, Manuel (1976), Libro de Apolonio. Estudios, ediciones y concordancias. Vol. I: Estudios. Madrid: Castalia, pp. 151-161.

Alvar, Manuel (1984), «Apolonio, clérigo entendido», en Symposium in honorem Prof. M. de Riquer. Barcelona: Quaderns Crema, pp. 63-65.

Ancos, Pablo (2018), «Encuentros y desencuentros de la Antigüedad tardía con la Edad Media en el Libro de Apolonio», Tirant, 21, pp. 281-300.

Artiles, Joaquín (1976), El «Libro de Apolonio», poema español del siglo XIII. Madrid: Gredos.

Berruezo SÁnchez, Diana (2012), «El conocimiento y la experiencia: dos formas de aprendizaje en el Libro de Apolonio», en Natalia Fernández Rodríguez y María Fernández Ferreiro (eds.), Literatura medieval y renacentista en España: líneas y pautas. Salamanca: SEMYR, pp. 381-386.

Biaggini, Olivier (2007), "“Todos somos romeros que camino pasamos": homo viator dans le mester de clerecía», Cahiers d'études hispaniques médiévales, 30, pp. 25-54.

Biglieri, Aníbal (2003), «Ciudad, campo y yermo en las vidas de santos de Gonzalo de Berceo», en Blanca Acinas Lopa (coord.), Studia Silensia XXVII. Silos un milenio. Actas del Congreso Internacional sobre la Abadía de Santo Domingo de Silos. Vol. 3: Cultura. Silos: Universidad de Burgos, pp. 539-554.

BrIFFAUD, Serge (1998), «De 1'“invention" du paysage. Pour une lecture critique des discours contemporains sur l'émergence d'une sensibilité paysagère en Europe», Comparaison: an international journal of comparative literature, II, pp. 35-56.

Collot, Michel (2014), Pour une géographie littéraire. Paris: Éditions de Minuit.

Corbella, Dolores (ed.) (2007), Libro de Apolonio [1992]. Madrid: Cátedra.

Crocoll, Natacha (2018), «El paisaje: estado de la cuestión y nuevas pistas de investigación», en Gaetano Lalomia y Daniela Santonocito (coords.), Literatura Medieval (Hispánica): nuevos enfoques metodológicos y críticos. San Millán de la Cogolla: Cilengua, pp. 167-176.

Crocoll, Natacha (2019), «Literary landscape in the Castilian Middle Ages: allegorical construction as a feature of textual landscapes», Journal of the LUCAS graduate conference, 7, pp. 94-106.

Cuesta Torre, María Luzdivina (1999), «La muerte aparente: un episodio del Libro de Apolonio», Livius, 13, pp. 9-21. 
Desing, Matthew (2011), «Luciana's story: text, travel, and interpretation in the Libro de Apolonio», Hispanic Review, 79/1, pp. 1-15.

Deyermond, Alan (1989), «Emoción y ética en el Libro de Apolonio», Vox Romanica, 48, pp. 153-164.

Gómez REDONDO, Fernando (2009), «Lectura y recepción en el Libro de Apolonio», en Antonio Chas Aguión y Cleofé Tato García (coords.), «Siempre soy quien ser solía»: Estudios de literatura española medieval en homenaje a Carmen Parrilla. A Coruña: Universidade da Coruña, pp. 163-184.

GonzÁlez VERA, José Luis (2001), «El mar en la poesía castellana medieval», Litoral, 232, pp.13-22.

LEBRERo COCHO, Jorge (2015), «Hidrofobia medieval: miedos y peligros vinculados al agua en la literatura castellana del XV», Medievalismo, 25, pp. 261-284.

Milani, Raffaele (2007), El arte del paisaje. Madrid: Biblioteca Nueva.

Navarro GonzÁlez, Alberto (1962), El mar en la literatura medieval castellana. Tenerife: Universidad de la Laguna, pp. 242-262.

Pascual Argente, Clara (2010), Visions of Antiquity. Remembering the classical past in the Castilian Roman Antique. [Tesis doctoral] Georgetown University, pp. 142-144.

RIBÉMONT, Bernard (2003), «Une géocritique de la littérature médiévale?», en Juliette Vion-Dury, Jean-Marie Grassin y Bertrand Westphal (dirs.), Littérature et espaces. Actes du XXX Congrès de la Société Française de Littérature Générale et Comparée. Limoges, 20-22 septembre 2001. Limoges: Presses Universitaires de Limoges, pp. 41-47.

Rodríguez Bote, María Teresa (2014), «La visión estética del paisaje en la Baja Edad Media», Medievalismo, 24, pp. 371-397.

Rodríguez Victoria, Rafael (2019), «La ciudad en el Libro de Apolonio», Medievalia, 51, pp. 71-84.

SCORDILIS BROWNLEE, Marina (1983), «Writing and scripture in the $\mathrm{Li}$ bro de Apolonio: the conflation of hagiography and romance», Hispanic Review, 51:2, pp. 159-174.

WeISs, Julian (2006), The 'mester de clerecia'. Intellectuals and ideologies in thirteenth-century Castille. London: Tamesis, pp. 198-209.

Zubillaga, Carina (2015), «Expectación narrativa y desarrollo de los acontecimientos en el Libro de Apolonio», eHumanista. Journal of Iberian Studies, 29, pp. 431-445. 
$\cos$

\section{ESPACIOS DE AVENTURAS EN EL LIBRO DE APOLONIO}

RESUMEN: El presente artículo explora las posibilidades de estudio abiertas por el examen del espacio en el Libro de Apolonio y su papel en la elaboración del discurso narrativo. A través del análisis textual de una serie de episodios concretos, se examina la función poética de los dos espacios que estructuran el relato, la ciudad y el mar, antes de observar las distintas lecturas simbólicas que estos tienen en el desarrollo de la acción y en la trayectoria de los distintos personajes.

Palabras-Clave: Espacio. Libro de Apolonio. Simbolismo. Estructura. Paisaje. Mar. Ciudad.

\section{SPACES OF ADVENTURE IN THE LIBRo DE APOLONIO}

ABSTRACT: This article explores the possibilities for research offered by the study of space in the Libro de Apolonio and its part in the elaboration of the narrative discourse. Through the textual analysis of a few concrete episodes, the poetic function of the two main spaces that structure the story, the town and the sea, will be considered. The reflexion will then focus on the various symbolic readings of the same spaces and the role that they play in the plot's development and in the different characters' path.

Keywords: Space. Libro de Apolonio. Symbolism. Structure. Landscape. Sea. Town. 